\title{
On admissible pairs and equivalent feedback-Youla parameterization in iterative learning control ${ }^{\text {th }}$
}

\author{
Mark Verwoerd $^{\mathrm{a}, *}$, Gjerrit Meinsma ${ }^{\mathrm{b}}$, Theo de Vries ${ }^{\mathrm{b}}$ \\ ${ }^{a}$ Hamilton Institute, National University of Ireland, Maynooth, Co. Kildare, Ireland \\ ${ }^{\mathrm{b}}$ Department of Electrical Engineering, Mathematics, and Computer Science, University of Twente, P.O. Box 217, 7500 AE Enschede, The Netherlands
}

Received 19 December 2003; received in revised form 26 May 2006; accepted 29 June 2006

Available online 1 September 2006

\begin{abstract}
This paper revisits a well-known synthesis problem in iterative learning control, where the objective is to optimize a performance criterion over a class of causal iterations. The approach taken here adopts an infinite-time setting and looks at limit behavior.

The first part of the paper considers iterations without current-cycle-feedback (CCF) term. A notion of admissibility is introduced to distinguish between pairs of operators that define a robustly converging iteration and pairs that do not. The set of admissible pairs is partitioned into disjoint equivalence classes. Different members of an equivalence class are shown to correspond to different realizations of a (stabilizing) feedback controller. Conversely, every stabilizing controller is shown to allow for a (non-unique) factorization in terms of admissible pairs. Class representatives are introduced to remove redundancy. The smaller set of representative pairs is shown to have a trivial parameterization that coincides with the Youla parameterization of all stabilizing controllers (stable plant case).

The second part of the paper considers the general family of CCF-iterations. Results derived in the non-CCF case carry over, with the exception that the set of equivalent controllers now forms but a subset of all stabilizing controllers. Necessary and sufficient conditions for full generalization are given.
\end{abstract}

(C) 2006 Elsevier Ltd. All rights reserved.

Keywords: Iterative learning control; Youla parameterization; Feedback control; $H_{\infty}$ control; Equivalent control; Linear systems

\section{Introduction}

Some 20 years ago, Arimoto, Kawamura, and Miyazaki (1984) were among the first to develop a theory of learning specifically tailored to single-loop control problems. Upon observing the human tendency to learn from experience, they were led to ask whether it would be possible to implement a similar ability in the automatic operation of dynamical systems. In answer, they proposed a 'betterment process', now known as iterative learning control (ILC). The method proved effective and inspired a great number of researchers. Over the years, Arimoto's original algorithm has been modified and

\footnotetext{
This paper was not presented at any IFAC meeting. This paper was recommended for publication in revised form by Associate Editor Gang Tao under the direction of Editor Miroslav Krstic.

* Corresponding author.

E-mail addresses: mark.verwoerd@nuim.ie (M. Verwoerd), g.meinsma@utwente.nl (G. Meinsma), t.j.a.devries@utwente.nl (T. de Vries).
}

extended in a number of ways: assumptions have been relaxed, robustness has been improved, and convergence properties have been laid out in detail. See Moore, Dahleh, and Bhattacharyya (1992) and Amann, Owens, and Rogers (1994) for an overview of early results. Today, there is an extensive literature covering a wealth of different learning rules applicable to a wide range of systems, both linear and nonlinear. Recent surveys include Moore (1999), Chen and Wen (1999), and Xu and Tan (2003).

In the early days, questions of analysis and synthesis were addressed almost exclusively within a time-domain framework which was built around the finite-trial-length postulate (Arimoto, 1998, chap. 1; Arimoto et al., 1984). This framework became the standard for many years and is still among the most commonly used today. Yet, over the course of two decades, a variety of other techniques have been introduced, some to considerable effect. This paper is about one such technique. The technique in question originates in the early nineties, when, following developments in the general field of control, people 
begin to view ILC as an $H_{\infty}$ synthesis problem. The synthesis problem is to minimize a performance criterion, typically the mean-squared tracking error, over the space of bounded (realrational) transfer functions, $R H_{\infty}$. See, for example, Padieu and Su (1990), Kavli (1992), Amann, Owens, Rogers, and Wahl (1996), and Moore et al. (1992). In this approach the finite-triallength postulate is dropped and an extra assumption introduced, namely that learning operators be causal (recall that every element in $R H_{\infty}$ defines a causal bounded (finite-dimensional) LTI operator). At the time, few would have anticipated that as natural a role as causality plays in conventional feedback control, as restrictive and unnecessary it would prove in the context of ILC. The success of Arimoto's learning rule was known to be tied up with the availability of a future error (i.e. with a non-causal ingredient of some kind). And indeed, the fact that a learning operator need not be causal was well-established (Moore et al., 1992; Moore, 1999). Yet it would seem that the precise reason as to why causality should affect ILC performance the ways it does was not known. In contrast, the implications for compensator design were well-understood, owing largely to the classical work of Bode. By the time Goldsmith $(2001,2002)$ introduced the notion of equivalent feedback, the fact that causality seriously impairs the achievable performance was widely acknowledged, and studies into the merits of noncausal ILC were well-underway. Indeed, the thesis that a noncausal approach would constitute 'the only viable route for ILC' (Goldsmith, 2002, p. 708) had been voiced by others before. It would appear however that Goldsmith was the first to provide compelling evidence for it. The evidence has been contested (see Owens \& Rogers, 2004; Goldsmith, 2004) but as of yet the thesis has not been overthrown. The work presented in this paper builds on that of Goldsmith's. We provideseveral extensions, most notably a converse result, which states that the set of equivalent controllers is generally but a subset of all stabilizing controllers. Conditions under which both sets coincide are given. Also, we state precise conditions (as captured by our notion of admissibility) under which causal ILC and conventional feedback are equivalent and provide an example of a causal iteration with an equivalent controller that is destabilizing.

Following Padieu and Su (1990), Kavli (1992), Moore et al. (1992), Moore (1993), de Roover (1996) and Amann et al. (1996), among others, this paper poses the problem of ILC as a two-parameter synthesis problem. The parameters are assumed causal bounded operators acting on the current input and current error, respectively. Our approach comprises the following steps. First, a notion of admissibility is introduced. This notion is used to single out 'bad' pairs of operators. Then the two-parameter problem is shown to be overparameterized; that is, different admissible pairs are shown to induce different sequences converging to the same fixed point. Redundancy is removed by grouping 'equivalent pairs' into equivalence classes, restricting attention to class representatives. Finally, the resulting one-parameter problem is shown to be a standard compensator design problem. The organization of the paper is as follows. Section 2 introduces the problem of ILC. Two problem cases are identified and discussed in subsequent sections: standard ILC in Section 3 and current-cycle-feedback-ILC (CCF-
ILC) in Section 4. Section 5 closes with conclusions and recommendations.

\section{Iterative learning control}

\subsection{Problem statement}

Let $U, Y$ be vector spaces. Given a plant $P: U \mapsto Y$, along with some $y_{\mathrm{d}} \in Y$, the problem of ILC is to construct a sequence $\left\{u_{i}\right\}$, with $u_{i} \in U$ for all $i$, such that $\left\{\left(u_{i}, P u_{i}\right)\right\}$ converges to a limit point in $U \times Y$ and $\bar{y}:=\lim _{i \rightarrow \infty} P u_{i}$ is close to $y_{\mathrm{d}}$. We shall assume that $P$ is LTI and finite dimensional, i.e. that $P(s)$ is real-rational. Throughout the paper, the space of inputs $U$ is $L_{2}[0, \infty)\left(H_{2}\right)$.

Let us consider an example. Let $P: L_{2}[0, \infty) \mapsto L_{2}[0, \infty)$ be defined as

$(P u)(t):=\int_{0}^{\infty} \mathrm{e}^{-(t-\tau)} u(\tau) \mathrm{d} \tau, \quad t \geqslant 0$

and let $y_{\mathrm{d}}$ be given as $y_{\mathrm{d}}:=P u_{\mathrm{d}}$, where

$u_{\mathrm{d}}:= \begin{cases}t(1-t) & \text { for } 0 \leqslant t<1 \\ 0 & \text { for } t \geqslant 1\end{cases}$

We select $u_{0}(t):=\mathrm{e}^{-t} \sin (t), t \geqslant 0$, and recursively define

$u_{k+1}(t):=u_{k}(t)+e_{k}(t), \quad k=0,1, \ldots$.

Here, $e_{k}(t):=y_{\mathrm{d}}-P u_{k}(t)$ denotes the current tracking error. Focusing on the system's output on the finite interval $[0,5]$ we are interested in the evolution of the mean-squared error (MSE), $\left\|e_{k}\right\|:=\frac{1}{5} \int_{0}^{5} \mathrm{e}_{k}^{2}(t) \mathrm{d} t$, as a function of the iteration number, $k$. Fig. 1 shows that the MSE progressively decreases, and does so in an exponential fashion. Also shown is the system's output after 10 trials, $y_{10}(t)$, along with the desired output $y_{\mathrm{d}}(t)$. This concludes the example.

We remark that system (3) may equivalently be defined as

$u_{k+1}(s):=u_{k}(s)+e_{k}(s), \quad k=0,1, \ldots$,

where $u_{k}(s)$ and $e_{k}(s)$ denote the images of $u_{k}(t)$ and $e_{k}(t)$ under the one-sided Laplace transform. In the sequel we shall adopt this so-called frequency-domain representation. Note that this is a matter of preference and does not encompass any loss of generality. In the remainder of the paper it is assumed that the sequence $\left\{u_{k}\right\}$ is generated by an element of the iteration class $T_{C}(Q, L): \mathrm{H}_{2} \rightarrow \mathrm{H}_{2}$,

$u_{k+1}:=Q u_{k}+L e_{k}+C e_{k+1}, \quad k=0,1, \ldots$

and that $Q$ and $L$ take values in $R H_{\infty}$. Here $e_{k}:=y_{\mathrm{d}}-y_{k}$ is the (current) tracking error we introduced earlier. The parameter $C$ represents a fixed feedback map and is not considered a design parameter. Lastly, the interconnection of $P$ and $C$ is assumed 

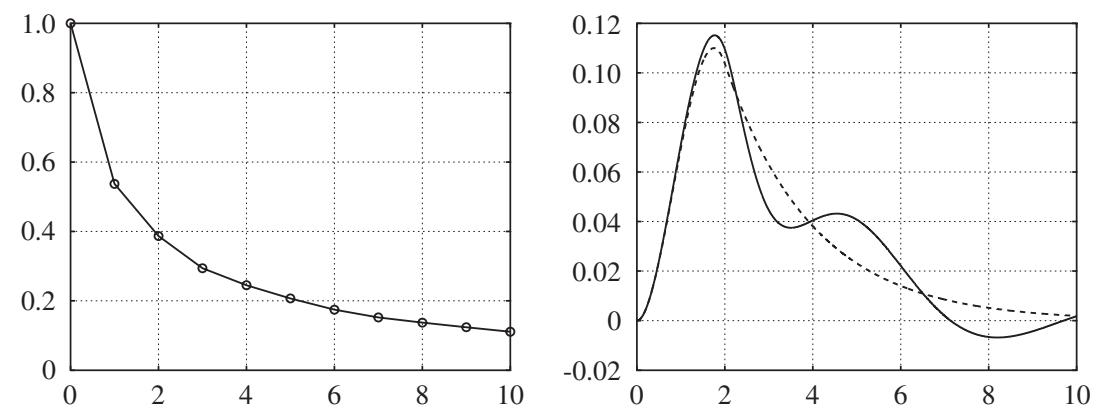

Fig. 1. Simulation results for the iterative system (3). The left plot depicts the mean-squared tracking error $\left\|e_{k}\right\|$, evaluated over the interval [0,5], and normalized against $\left\|e_{0}\right\|, k=0,1, \ldots, 10$; the plot on the right shows the output of the plant after 10 trials, $y_{10}(t)$, along with the desired output $y_{\mathrm{d}}(t)$ (dashed).

internally stable, i.e. $(P, C)$ is assumed to satisfy

$\left[\begin{array}{cc}(I+C P)^{-1} & -C(I+P C)^{-1} \\ P(I+C P)^{-1} & (I+P C)^{-1}\end{array}\right] \in R H_{\infty}$.

Within this framework, we address the following questions:

(1) What are the pairs $(Q, L)$ for which (5) defines a robustly converging sequence of input-output pairs?

(2) What is the interplay between $Q$ and $L$; how do these parameters, separately or jointly, determine asymptotic performance and convergence speed?

(3) How must one choose $(Q, L)$ so that small asymptotic error results?

We emphasize that these issues are not all new. In particular, the literature associated with the synthesis problem (3) is quite extensive. However, the present treatment appears to be of independent interest in that it exploits the concept of equivalent feedback to reinterpret existing results from a conventional feedback point of view, and in so doing provides new insight in the ILC synthesis problem. In subsequent sections, we present our findings for the standard ILC problem (Section 3) and the more general CCF-ILC problem (Section 4). This section concludes with a well-posedness lemma.

Lemma 1. Consider the class of recurrences (5). Let $y_{\mathrm{d}} \in \mathrm{H}_{2}$. Suppose $(P, C)$ satisfies (6). For all $Q, L \in R H_{\infty}$ we have that if $\left(u_{k}, y_{k}\right) \in H_{2} \times H_{2}$ then $\left(u_{k+1}, y_{k+1}\right) \in H_{2} \times H_{2}$.

Proof. Suppose $\left(u_{k}, y_{k}\right) \in H_{2}$. Define $z_{k}:=Q u_{k}+L e_{k}$. With $Q, L \in R H_{\infty}$, it follows that $z_{k} \in H_{2}$. To compute $\left(u_{k+1}, y_{k+1}\right)$, we solve

$u_{k+1}=z_{k}+C\left(y_{\mathrm{d}}-y_{k+1}\right)$,

$y_{k+1}=P u_{k+1}$,

the solution of which is given by

$u_{k+1}=(I+C P)^{-1} z_{k}+(I+C P)^{-1} C y_{\mathrm{d}}$,

$y_{k+1}=P(I+C P)^{-1} z_{k}+P(I+C P)^{-1} C y_{\mathrm{d}}$.

By internal stability all four transfer matrices are stable. Thus, with $z_{k}, y_{\mathrm{d}} \in H_{2}$, it follows that $u_{k+1}, y_{k+1} \in H_{2}$. This concludes the proof.

\section{The standard ILC problem}

\subsection{Introduction}

In what we shall term the standard ILC problem, $P$ is assumed stable and $C$ is set to zero $(C=0)$. Under these conditions, Eq. (5) simplifies to

$u_{k+1}:=Q u_{k}+L e_{k}, \quad k=0,1, \ldots$.

This particular class of iterations has received considerable attention. Moore et al. (1992) studied its convergence properties and derived an upper bound on the achievable performance. Kavli (1992) discussed robustness and derived a synthesis procedure for selecting the learning parameters and applied it to the control of a robot manipulator. de Roover (1996) improved on the available heuristic design procedures by deploying $H_{\infty}$ optimization techniques. See also Amann et al. (1996).

Overall there is good agreement on how to choose the respective design parameters; the consensus is that $L$ should approximate the inverse of $P$ while $Q$ should compensate for the mismatch (which is typically greatest at high frequencies). At the same time, it appears that the interplay between the two parameters is not fully understood. The common argument is that to attain good performance $Q$ ought to be 'close to unity' in some appropriate frequency band (for technical reasons owing to causality, $Q$ cannot be equal to unity for all frequencies). In a typical situation, $Q$ would have low-pass characteristics so as to ensure good tracking at low frequencies (where $Q$ is close to unity). With $Q$ fixed, $L$ is then 'matched' to the inverse of the given plant by solving what is essentially a model matching problem de Roover (1996). This two-step approach, which is common to many procedures, appears to lack proper justification. By fixing one of the parameters, the design problem is simplified at the expense of generality. In contrast, the results in the next section will show that the problem of ILC can be reduced to a one-parameter problem without loss of generality. Again, this observation is not new: an early paper by Moore et al. (1992) contains essentially the same idea. However, there it appears as an isolated result without clear interpretation, whereas here the result has a natural explanation in terms of equivalent feedback. 


\subsection{On admissibility}

The family of iterations (9) comprises a mixture of convergent and non-convergent elements. This suggests that the set of pairs $(Q, L)$ can be partitioned into two subsets made up of, respectively, admissible and non-admissible pairs. To make this notion precise, consider a class of perturbed iterations

$u_{k+1}^{w}:=Q u_{k}^{w}+L \mathrm{e}_{k}^{w}+w_{k}, \quad k=0,1, \ldots$.

We shall assume that $w_{k}$ takes values in $H_{2}$. Note that this family is identical to that defined in (9), except for the perturbation term $w_{k}$; this term will serve to impose a continuity constraint on the solution of the unperturbed iteration. That is, assuming $u_{0}^{w}=u_{0}$, solutions of (9) and (10) are required to be arbitrarily close for small enough $\left\|w_{k}\right\|$.

Definition 2. Given $P \in R H_{\infty}$. Consider the family of perturbed iterations (10). Let $\left\{u_{k}^{0}\right\}$ denote the unique unperturbed solution corresponding to $w \equiv 0$. The pair $(Q, L) \in R H_{\infty}^{2}$ is said to be admissible if:

(1) for every $y_{\mathrm{d}} \in H_{2}$ there exists $\bar{u}\left(y_{\mathrm{d}}\right) \in H_{2}$ such that $\lim _{k \rightarrow \infty} u_{k}^{0}=\bar{u}\left(y_{\mathrm{d}}\right)$ for every $u_{0}^{0} \in H_{2}$;

(2) for every $\varepsilon>0$, there exists $\delta>0$ such that whenever $\sup _{k}\left\|w_{k}\right\|_{H_{2}}<\delta$ then $\sup _{k}\left\|u_{k}^{w}-u_{k}^{0}\right\|_{H_{2}}<\varepsilon$.

The set of all admissible pairs will be denoted by $A$.

In this paper, the notion of admissibility shall play a role ana$\log$ to that of internal stability in feedback control. Its purpose is to single out 'bad' pairs of operators. We remark that our definition is restrictive in that it insists on convergence over infinite time in contrast with other approaches, which frequently employ notions of convergence defined over finite time. To appreciate the difference between these two types of convergence, let us introduce the family of operators $\Gamma_{\lambda}:[0, T] \mapsto[0, T]$

$\left(\Gamma_{\lambda} u\right)(t):=u(t) \mathrm{e}^{-\lambda t}, \quad t \in[0, T]$.

Here, $\lambda$ takes values in $\mathbb{R}_{+}$. The parameter $T$ can take any positive real value (up to and including $\infty$ ), depending on the domain of definition of $u$. We make the following observation: for all finite $T$ and all $\lambda>0$ we have that $\Gamma_{\lambda} u \in L_{2}[0, T]$ iff $u \in L_{2}[0, T]$. This implies that if $\left\{\Gamma_{\lambda} u_{k}\right\}$ has a limit point, then so has $\left\{u_{k}\right\}$. Note that when $T=\infty$, this is no longer true and as it turns out, this is the essential difference between finite- and infinite-time convergence. Now let $G$ be a stable LTI operator defined on $[0, \infty)$ and let $G_{[0, T]}$ denote the restriction of $G$ to $[0, T]$, that is,

$\left(G_{[0, T]} u\right)(t):=(G u)(t), \quad t \in[0, T]$

for all $u$. Suppose $u_{0} \in L_{2}[0, T]$ and recursively define $u_{k}$, as follows:

$u_{k+1}(t):=G_{[0, T]} u_{k}, \quad k=0,1, \ldots$.

Using basic properties of the Laplace transform and a contraction-type argument, it can be shown that a sufficient condition for $\left\{\Gamma^{\lambda} u_{k}\right\}$ (and thus also for $\left\{u_{k}\right\}$ ) to have a limit point in $L_{2}[0, T]$ is that

$$
\sup _{\operatorname{Re}(s)>0} \bar{\sigma}(G(s+\lambda))<1,
$$

for some $\lambda>0$. Hence, it follows that a sufficient condition for $\left\{u_{k}\right\}$ to converge is that

$\bar{\sigma}(G(\infty))<1$,

a condition that is considerably weaker than its infinite-time counterpart,

$\sup _{\omega \in \mathbb{R}} \bar{\sigma}(G(\mathrm{j} \omega))<1$

(Theorem 3). We shall nonetheless insist on using infinite-time convergence as a condition for admissibility. The next theorem gives a necessary and sufficient condition for a pair $(Q, L)$ to be admissible in the sense of Definition 2.

Theorem 3. Given the triple $(P, Q, L) \in R H_{\infty}^{3}$. The pair $(Q, L)$ is admissible in the sense of Definition 2 if and only if $\|Q-L P\|_{\infty}<1$.

Proof. The proof of sufficiency follows standard contraction arguments and can essentially be found in e.g. Moore et al. (1992) and Padieu and $\mathrm{Su}$ (1990). We prove necessity, focusing on the SISO case. Define $F:=Q-L P, d:=L y_{\mathrm{d}}$ and rewrite (10) to obtain

$u_{k+1}^{w}=F u_{k}^{w}+d+w_{k}$.

Suppose $\|F\|=1$ (the case $\|F\|>1$ is easy). What we need to show is that for every $\delta>0$ and every $\varepsilon>0$ there exists $K>0$ such that for at least some $u_{0}^{w}$ and some $y_{\mathrm{d}}$, there is a sequence $\left\{w_{k}\right\}$ with $\left\|w_{k}\right\|<\delta, k \leqslant K-1$, such that $\left\|u_{K}^{w}-u_{K}^{0}\right\|>\varepsilon$. Let $y_{\mathrm{d}}=u_{0}^{w}=0$, so that $u_{k}^{0} \equiv 0$. Given $K$, we construct $w$ such that $\left\|F^{K} w\right\| \geqslant \frac{1}{2}\|w\|$.

The fact that (for SISO systems) $\left\|F^{K}\right\|=\|F\|^{K}=1$ implies that such $w$ exists; this follows by continuity of the norm. Define $w_{k}:=F^{k} w, k=0,1, \ldots, K-1$. It follows that $u_{k}^{w}=k F^{k} w$ and by construction $\left\|u_{K}^{w}\right\| \geqslant \frac{1}{2} K\|w\|$. Choose $w$ such that $\|w\|=\delta / 2$ and take $K=\lceil 4(\varepsilon / \delta)\rceil+1$. Then $\left\|u_{K}^{w}-u_{K}^{0}\right\|=\left\|u_{K}^{w}\right\|>\varepsilon$. This concludes the proof.

The next lemma reveals a property of admissible pairs that will be useful in subsequent analysis.

Lemma 4. Let $P \in R H_{\infty}$. For any $(Q, L) \in A$, the pair $((I-Q), L)$ is left-coprime, and, as a consequence, the fraction $(I-Q)^{-1} L$ cannot have unstable pole-zero cancellations. Moreover, if $P$ is strictly proper then $(I-Q)^{-1} L$ is a proper transfer matrix.

Proof. Recall that two transfer matrices $(I-Q)$ and $L$ in $R H_{\infty}$ are left-coprime over $R H_{\infty}$ iff there exist matrices $X$ and $Y$ in $R H_{\infty}$ such that

$(I-Q) X+L Y=I$. 
Define $X=(I-Q+L P)^{-1}, Y=P X$. Note that $X \in R H_{\infty}$ by assumption of admissibility ( $\|Q-L P\|_{\infty}<1$, see Theorem 3) and hence also $Y \in R H_{\infty}$. Clearly, $(I-Q) X+L Y=I$, which proves left-coprimeness. Now suppose $P$ is strictly proper, i.e. $P(\infty)=0$. Then, at infinity, $(I-Q+L P)^{-1}$ reduces to $(I-$ $Q)^{-1}$, since $L(\infty) P(\infty)=0$. Moreover since $(I-Q+L P)^{-1}$ is bounded at infinity, so is $(I-Q)^{-1}$. From this we conclude that the composite transfer matrix $(I-Q)^{-1} L$ is proper.

We shall now temporarily discontinue our discussion in order to review some results related to the notion of equivalent feedback.

\subsection{Causal ILC and equivalent feedback control}

The idea of equivalent feedback for ILC has been discussed in a series of recent papers (Goldsmith, 2001, 2002; Verwoerd, Meinsma, \& de Vries, 2002, 2003). The main thesis communicated in these papers is that (causal) ILC and conventional feedback control are essentially equivalent. This equivalence is best understood by considering the family of iterations (9),

$u_{k+1}:=Q u_{k}+L e_{k}, \quad k=0,1, \ldots$.

Assuming convergence $\left(\lim _{k \rightarrow \infty} u_{k}=u ; \lim _{k \rightarrow \infty} e_{k}=e\right)$, it is easy to see that in the limit, the pair $\left(u_{k}, e_{k}\right)$ satisfies

$(I-Q) u=L e$,

which is equivalent to

$u=(I-Q)^{-1} L e$

provided $(I-Q)^{-1} L$ is well-defined and proper-a fact we established in Section 3.2 (Lemma 4). Eq. (21) specifies a relation between the control input $u$ and the tracking error $e$ and can be interpreted as a defining equation for the feedback controller $K=(I-Q)^{-1} L$ (see Fig. 2). Note that this controller is expressed in terms of the parameters $Q$ and $L$. Hence, its construction requires no prior knowledge (other than what was required to pick the ILC parameters). More importantly, it follows that this compensator would return the same performance without the need to engage in a process of iteration (Goldsmith, 2002). A detailed discussion of this result and its implications is deferred until the end of this section. Formally, the equivalent controller is defined as follows:

Definition 5. Given $P \in R H_{\infty}$ strictly proper. For every $(Q, L) \in A$ we define the equivalent controller

$K:=(I-Q)^{-1} L$

Not unexpected, the equivalent controller is internally stabilizing.

Theorem 6. Let $P \in R H_{\infty}$ be strictly proper and let $(Q, L)$ be admissible. Then the equivalent controller (22) is internally stabilizing.

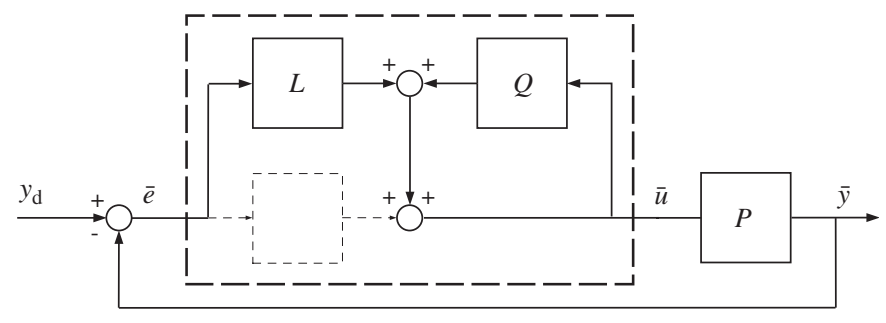

Fig. 2. Equivalent feedback controller (fat dashed box) for standard ILC.

Proof. Let $\tilde{V}^{-1} \tilde{U}$ and $N M^{-1}$ be left- and right-coprime factorizations of the controller $K$ and the plant $P$, respectively. Then Zhou, Doyle, and Glover (1996, Lemma 5.1) say that $K$ internally stabilizes $P$ if and only if

$(\tilde{V} M+\tilde{U} N)^{-1} \in R H_{\infty}$.

We substitute $\tilde{V}=(I-Q), \tilde{U}=L, M=I$, and $N=P$ into condition (23), which then evaluates to

$(I-Q+L P)^{-1} \in R H_{\infty}$.

This condition is satisfied by assumption of admissibility (see Theorem 3), where we use the fact that for every $(Q, L) \in A$, $(I-Q)$ and $L$ are left-coprime (Lemma 4).

The following theorem states that every stabilizing controller admits a parameterization in terms of admissible pairs.

Theorem 7. Let $P \in R H_{\infty}$ be strictly proper, and let $K$ be a stabilizing controller for $P$. Then there exists $(Q, L) \in A$ such that $(I-Q)^{-1} L=K$.

Proof. For any $V \in R H_{\infty}$, the unique solution $\left(Q_{V}, L_{V}\right)$ to the set of equations

$\left.\begin{array}{l}Q_{V}-L_{V} P=V \\ L_{V}=\left(I-Q_{V}\right) K\end{array}\right\}$

is given by

$\left.\begin{array}{l}Q_{V}=I-(I-V)(I+K P)^{-1} \\ L_{V}=(I-V) K(I+P K)^{-1} .\end{array}\right\}$

By assumption of internal stability, the closed-loop transfer functions $(I+K P)^{-1}$ and $K(I+P K)^{-1}$ are both stable. Hence, we conclude $Q_{V}, L_{V} \in R H_{\infty}$. Now let $V$ be a contraction, i.e. $\|V\|_{\infty}<1$. Then, by construction, $\left(Q_{V}, L_{V}\right) \in A$ (Theorem $3)$.

The proof of Theorem 7 shows that different admissible pairs may define one and the same equivalent controller. This suggests that such pairs can be considered equivalent. In the next section we will formalize this observation as we introduce an equivalence relation on the set of admissible pairs. 


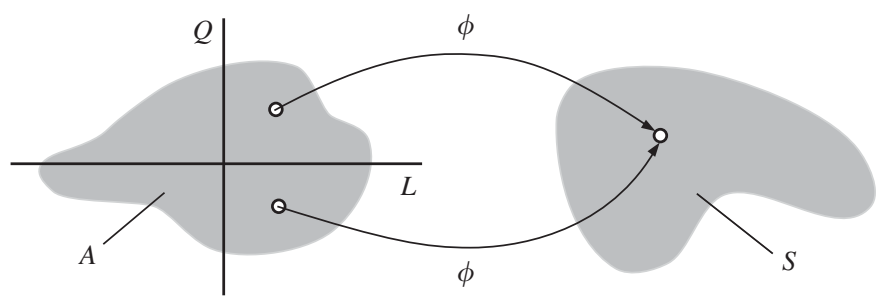

Fig. 3. The map $\phi: A \mapsto S$ is surjective but not injective.

\subsection{On equivalence and minimal parameterization}

Definition 8. Let $P \in R H_{\infty}$ be strictly proper and let $A$ be the set of admissible pairs. Two elements $\left(Q_{1}, L_{1}\right),\left(Q_{2}, L_{2}\right) \in A$ are said to be equivalent if they satisfy the equivalence relation

$\left(I-Q_{1}\right)^{-1} L_{1}=\left(I-Q_{2}\right)^{-1} L_{2}$.

We remark that strict properness is imposed for convenience only. Without this assumption, the matrix fraction $(I-Q)^{-1} L$ need not be well-defined for all $(Q, L) \in A$. A more general condition would be

$\left(I-Q_{1}+L_{1} P\right)^{-1} L_{1} P=\left(I-Q_{2}+L_{2} P\right)^{-1} L_{2} P$.

The drawback of this condition is that it involves the unknown plant parameter $P$, which makes it hard to test for equivalence. From an ILC point of view, equivalence in the sense of Definition 8 corresponds to different iterations converging to the same fixed point $\bar{u} \in \mathrm{H}_{2}$,

$$
\begin{aligned}
\bar{u} & =(I-Q+L P)^{-1} L y_{\mathrm{d}} \\
& =(I+\underbrace{(I-Q)^{-1} L}_{K} P)^{-1} \underbrace{(I-Q)^{-1} L}_{K} y_{\mathrm{d}} .
\end{aligned}
$$

Eq. (27) reveals that the fixed point is completely determined by the fraction $(I-Q)^{-1} L$. In Section 3.3, the matrix fraction $(I-Q)^{-1} L$ appeared as a left-coprime factorization of the equivalent controller. So from a controller synthesis point of view, equivalent admissible pairs correspond to different leftcoprime factorizations of the same equivalent controller. To further investigate the relation between standard ILC and conventional feedback control, let us introduce the set $S$ of all stabilizing controllers corresponding to a given plant $P$, along with the map $\phi: A \mapsto S$,

$K=\phi(Q, L):=(I-Q)^{-1} L$.

By Theorems 6 and 7 we know that the map $\phi$ is many-to-one and onto, i.e. surjective but not injective (see Fig. 3). By considering equivalence classes instead, we can uniquely identify every element $K \in S$ with a class

$[(\hat{Q}, \hat{L})]:=\{(Q, L) \in A:(Q, L) \simeq(\hat{Q}, \hat{L})\}$.

This is illustrated in Fig. 4.

Still, we are associating elements in $S$ with sets in $A$. In order to create a map that is truly one-to-one, we restrict attention

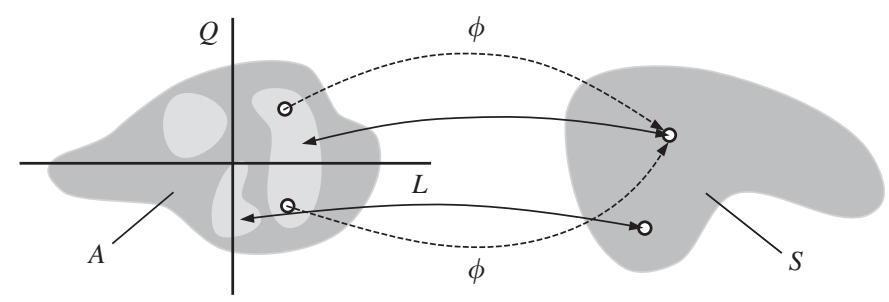

Fig. 4. Every equivalence class in $A$ allows for unique identification with a single stabilizing controller.

to class representatives. Two remarks are in order. First, by restricting attention to just one element (pair) in the class we do not lose generality. After all, pairs within a class are equivalent and by focusing on one element we effectively remove redundancy; second, the choice of representatives is, in principle, arbitrary. In this paper we choose to select as a representative for a given class $[(Q, L)]$ the element $\left(Q_{0}, L_{0}\right) \in[(Q, L)]$ that satisfies $Q_{0}-L_{0} P=0$. The following lemma states that every class has precisely one such member.

Lemma 9. Let $(Q, L) \in A$ be an admissible pair and let $[(Q, L)] \subset A$ denote the corresponding equivalence class. There exists a unique admissible pair $\left(Q_{0}, L_{0}\right) \in[(Q, L)]$ such that

$Q_{0}-L_{0} P=0$

Proof. Immediate from the proof of Theorem 7 (take $V=0$ ).

In the standard ILC problem, the quantity $Q-L P$ determines the convergence speed of the algorithm; the 'smaller' $Q-L P$ is, the faster convergence is achieved. Setting $Q_{0}-L_{0} P=0$ causes recursion (9) to converge within a single iteration step. Note that even though the location of the fixed point and the speed of convergence both depend on the same parameter pair $(Q, L)$ they are nonetheless independent. That is to say, any fixed point (within the reachable set induced by the admissible pairs) can be reached at any rate of convergence. This, essentially, is what Theorem 7 tells us.

We define $A_{0}$ to be the set of all class representatives or equivalently, the set of all pairs $(Q, L) \in A$ satisfying $Q-$ $L P=0$. Note that by definition every member of this set has the special form $(L P, L)$. Note furthermore that $A_{0}$ cannot contain any equivalent pairs since the representatives are taken from disjoint sets. As a consequence, the restriction of $\phi$ to $A_{0}$, is injective. Clearly, $\phi_{0}$ is also surjective and hence we conclude that $\phi_{0}: A_{0} \mapsto S$ is a bijection. In other words, the sets $A_{0}$ and $S$ are identical up to an isomorphism. This fact allows us to prove a well-known result.

Corollary 10 (Youla). Let $P \in R H_{\infty}$ be strictly proper. The set $S$ of all (proper real-rational) controllers $K$ stabilizing $P$ is given as

$S=\left\{(I-L P)^{-1} L: L \in R H_{\infty}\right\}$. 


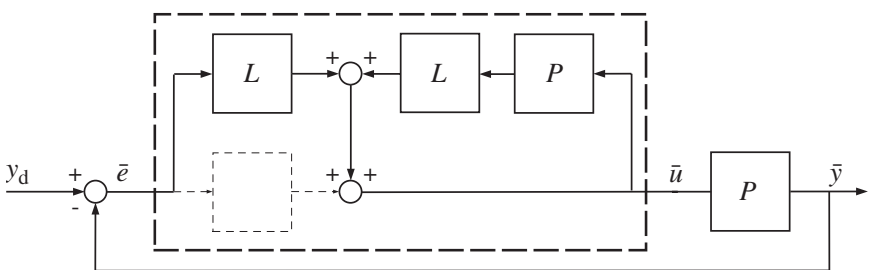

Fig. 5. Minimal parameterization of all stabilizing controllers. Shown is the equivalent controller (fat dashed box) under the constraint $Q=L P$. Compare with Fig. 2.

Proof. Note that the set of class representatives $A_{0}$ has a trivial parameterization

$A_{0}=\left\{(L P, L): L \in R H_{\infty}\right\}$

and recall that $A_{0}$ and $S$ are bijective under $\phi_{0}$. Hence, a parameterization of $A_{0}$ is also a parameterization of $S$. In particular

$$
\begin{aligned}
S & =\left\{\phi_{0}(L P, L): L \in R H_{\infty}\right\} \\
& =\left\{(I-L P)^{-1} L: L \in R H_{\infty}\right\} .
\end{aligned}
$$

This completes the proof.

Let us remark that it is again not necessary to impose strict properness. The problem with e.g. biproper plants however is that the fraction $(I-L P)^{-1} L$ need not be defined for all $L \in$ $R H_{\infty}$, as, for instance, in the case when $P$ is bistable and $L=$ $P^{-1}$. We could sidestep the issue by stating that ' $(I-L P)^{-1} L$ is a stabilizing controller whenever defined'. Instead we choose to insist on strict properness, which automatically warrants the fraction to be well-defined for all $L$. We point out that the resulting parameterization is minimal (i.e. involves a minimal number of parameters). The corresponding block diagram is given in Fig. 5.

The results in this section suggest that the standard ILC problem is equivalent to a compensator design problem for a stable plant. A similar finding was reported earlier in Phan, Longman, and Moore (2000, Observation 5) and Goldsmith (2002). It is important to note that this equivalence is, first and foremost, a theoretical result. That is to say, in theory we can map any compensator $C$ to an admissible pair $(Q, L)$ through application of the inverse map $\phi_{0}^{-1}: S \mapsto A_{0}$,

$$
\left(\begin{array}{c}
Q \\
L
\end{array}\right)=\left(\begin{array}{c}
K P(I+K P)^{-1} \\
(I+K P)^{-1} K
\end{array}\right)
$$

(compare with Eq. (26)). Note however that the inverse map (33) depends on the plant parameter $P$. This is true, not just for this specific map, but in general. To see this, let $P$ be a stable plant and let $C$ be a stabilizing controller for $P$. Then for any left-coprime pair $(U, V)$ such that $C=V^{-1} U$, we have that $V I-U P$ is invertible (has a stable inverse). Define $Q:=I-V$, $U:=L$. It follows that $I-(Q-L P)$ is invertible. The essence of the problem is that this condition, viz. that $I-(Q-L P)$ is invertible, is necessary and sufficient for closed-loop stability, but only necessary for admissibility. That is to say, even if we know that $I-(Q-L P)$ is invertible, additional knowledge about $P$ is required to conclude that the iterative scheme will converge. This observation notwithstanding, one can still bring to bear particular compensator design techniques. Consider, for example, the problem of designing an ILC scheme with small asymptotic error. In terms of compensator design criteria this amounts to shaping the output sensitivity function $S=(I+$ $P K)^{-1}$ such that $\bar{e}=S y_{\mathrm{d}}$ is small in an appropriate sense (e.g. within the bandwidth of interest). It follows from Corollary 10 that $\bar{e}$ can be expressed in terms of the free parameter $L \in$ $R H_{\infty}$ as follows:

$\bar{e}=(I-P L) y_{\mathrm{d}}$.

Designing for small $\bar{e}$ thus boils down to determining a good approximate inverse of $P$, see also Zames (1981) and Moore (1993). For the sake of the argument suppose $P$ is a SISO minimum-phase plant. We choose $L=Z P^{-1}$ (and $Q=Z$ ) where $Z$ is low-pass and has sufficient relative degree to ensure that $L$ is proper. This coincides with the design procedures discussed in Moore (1993) and de Roover (1996).

Given this connection, an obvious question to ask is why one would implement a causal ILC scheme knowing that a simple feedback controller would do. Indeed, there appears to be no good reason at all. At the same time one may recall that the findings presented in this paper rely on a rather specific notion of convergence, namely convergence over infinite time. When we allow for finite-time convergence, some results may fail to hold. To see this, let $Q, L$ be bounded causal linear operators as before. Consider the iterative system

$u_{k+1}:=Q_{[0, T]} u_{k}+L_{[0, T]} e_{k}, \quad k=0,1, \ldots$.

As discussed in Section 3.2, a sufficient condition for the system (35) to converge to a fixed point $\bar{u} \in L_{2}[0, T]$ is that $\bar{\sigma}(Q(\infty)-L(\infty) P(\infty))<1$. Let us now redefine admissibility to mean that a pair $(Q, L)$ satisfies this latter condition. To see if, with this new definition of admissibility, the result of Theorem 6 (admissible pairs define stabilizing controllers) still holds, consider the following numerical example. Let $P$ be given as $P(s):=(s-1) /(s+1)^{2}$ and define $Q(s):=1-\alpha$, $L(s):=\beta$. Provided $0<\alpha<2$, it follows that the pair $(Q, L)$ is admissible for all finite $\beta$. Inspection of the 'equivalent controller' $K=(I-Q)^{-1} L=(\beta / \alpha)$ shows that $K$ is stabilizing if and only if $|\beta / \alpha|<4$. Hence, there exist parameter combinations $(\alpha, \beta)$ that render the pair $(Q, L)$ admissible yet for which the equivalent controller fails to be stabilizing.

One could argue that what we are doing here is comparing the performance of ILC on a finite-time interval with that of feedback control on an infinite-time interval, which is not particularly fair. Against that we would argue that, if unfair, the comparison is nonetheless meaningful, if only because engineering practice demands that a feedback controller be stabilizing and for good reason at that. For one, if the feedback controller were destabilizing, divergence would occur within a single trial. In ILC, divergence is a much more gradual process. Thus, what this example ultimately demonstrates is that ILC provides a relatively safe method of getting that extra bit of performance.

Lastly, we point out that in most cases when an LTI operator $G$ is unbounded on $[0, \infty)$, its restriction $G_{[0, T]}$ is bounded on 
$[0, T]$. In an infinite-time setting, operators of the latter kind are clearly not to be considered admissible. In a finite-time setting, however, the issue is debatable; one may choose not to include such operators, but the reason (their gain, though finite, being very large) would be less compelling.

\section{CCF-ILC}

This section deals with the CCF-ILC problem. The obvious distinction between this and the standard ILC problem (Section 3 ) is the presence of a CCF term $C e_{k+1}$. Also, the plant is no longer assumed stable and hence, for reasons of well-posedness, $C$ is assumed stabilizing.

We shall consider the following two problems. Let $P$ be a proper real-rational plant, and let $C$ be a corresponding stabilizing controller $C$;

(1) let $(Q, L) \in A$ be admissible with respect to the family of iterations (5) and define the equivalent controller $K=$ $(I-Q)^{-1}(L+C)$ : Is $K$ always stabilizing?

(2) Given any stabilizing controller $K$, does there always exist an admissible pair $(Q, L) \in A$ for which $K$ is an equivalent controller?

As we will see, the respective answers are 'Yes' and 'No'. Note that for standard ILC both answers would have been affirmative (compare Theorems 6 and 7). We remark that the answer to the second question critically depends on $C$. This dependence will be explored in depth. Both questions and their respective answers are of interest. The first because it tells us that an equivalent stabilizing feedback controller always exists; the second because it advises us that would we decide to do ILC nonetheless the controller $C$ should be considered a design parameter.

\subsection{Admissibility and equivalent feedback}

For admissibility in the CCF-ILC case we adopt the same definition (Definition 2) as for the standard ILC case. The only difference to account for is that the initial condition $u_{0}$ is not free in $H_{2}$ due to smoothness constraints imposed by the closed loop. For this reason we introduce the auxiliary variable $\hat{u}_{0}$ (Fig. 6) and redefine admissibility with respect to this new initial input.

Consider the class of perturbed iterations:

$u_{k+1}^{w}=Q u_{k}^{w}+L \mathrm{e}_{k}^{w}+C \mathrm{e}_{k+1}^{w}+w_{k}, \quad k=0,1, \ldots$,

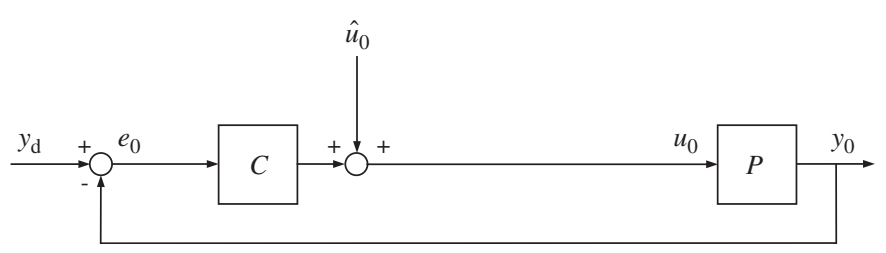

Fig. 6. Setting the initial condition in CCF-ILC. with initial input $u_{0}^{w}\left(\hat{u}_{0}\right)$. A pair $(Q, L) \in R H_{\infty}^{2}$ is said to be admissible if the respective sequences $\left\{u_{k}^{w}\right\}$ and $\left\{y_{k}^{w}\right\}$ are bounded under all bounded perturbations $\left(\sup _{k}\left\|w_{k}\right\|_{H_{2}}<\delta\right)$, and jointly approach the fixed point $(\bar{u}, \bar{y})$ of the unperturbed iteration (5) in a continuous fashion as the perturbation term subsides (compare Definition 2). Naturally, this should hold for all $\hat{u}_{0}$ and all $y_{\mathrm{d}}$.

We remark that the notion of admissibility is not to be confused with that of 'internal stability for ILC' Goldsmith (2002, Definition 1). To recall, iteration (5) is said to be internally stable if, in the presence of bounded disturbances and noise, both $e_{k}$ and $u_{k}$ remain bounded for all $k<\infty$. In our framework, internal stability for ILC follows from the assumption that $C$ is internally stabilizing and $Q, L$ are stable. Internal stability for ILC guarantees well-posedness (see Lemma 1), but the notion of admissibility is stronger: starting from a family of well-posed (internally stable) iterations, operators (and the corresponding family members) are classified into one of two subsets, depending on whether they have or lack certain convergence properties. We have the following theorem.

Theorem 11. Let $P$ be a real-rational plant (not necessarily stable), and let $C$ be a stabilizing controller. Consider the family of iterations (5). The pair $(Q, L)$ is admissible if and only if

$\left\|(Q-L P)(I+C P)^{-1}\right\|_{\infty}<1$.

Proof. Again, we prove only necessity. The proof runs along the same lines as that of Theorem 3. The idea is to consider the term $z_{k}:=Q u_{k}^{w}+L \mathrm{e}_{k}^{w}$, which may be viewed as 'the ILC contribution' to the feedback loop. If we can show that $\left\{z_{k}\right\}$ is bounded then, by internal stability, boundedness of $\left\{u_{k}\right\}$ and $\left\{y_{k}\right\}$ follow. Conversely, if we can construct $\left\{w_{k}\right\}$ and pick $K$, such that $\left\|z_{K}\right\|$ can be made arbitrarily large, it follows that either $\left\|u_{K}^{w}\right\|$ or $\left\|y_{K}^{w}\right\|$ can grow without bound, which would violate the condition for admissibility.

Define $F:=(Q-L P)(I+C P)^{-1}$ and verify that $z_{k}$ satisfies

$z_{k+1}=F z_{k}+F w_{k}+(F C+L) y_{\mathrm{d}}$.

Note that $(F C+L) \in R H_{\infty}$. With $d:=(F C+L) y_{\mathrm{d}}$ and $\tilde{w}_{k}:=F w_{k}$, Eq. (38) simplifies to

$z_{k+1}=F z_{k}+\tilde{w}_{k}+d$

which resembles Eq. (17). Let us observe that switching from $w_{k}$ to $\tilde{w}_{k}$ does not affect the construction used in the proof for the standard ILC case (Theorem 3). The rest of the proof would be a mere duplication and is thus omitted.

The next theorem shows that the equivalent controller for CCF-ILC is internally stabilizing.

Theorem 12. Given $P$ strictly proper and let $(Q, L)$ be an admissible pair for the family of CCF-ILC iterations defined in (5). Then the equivalent controller $K=(I-Q)^{-1}(L+C)$ defines a proper matrix transfer function. Moreover, the corresponding feedback system (Fig. 7) is well-posed and internally stable. 


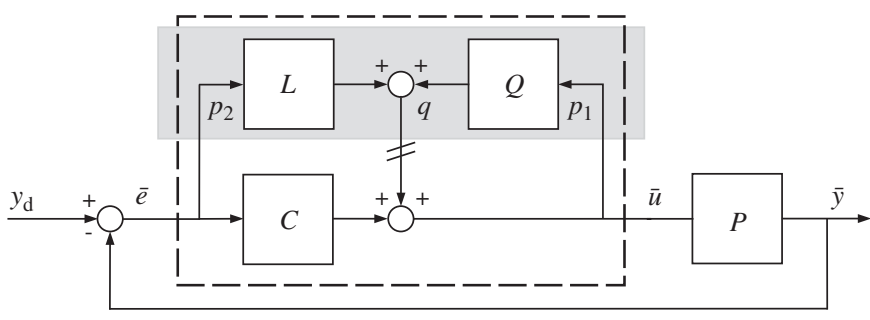

Fig. 7. Equivalent feedback controller (dashed) and the ILC-subsystem (shaded) for CCF-ILC.

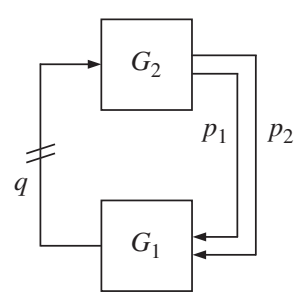

Fig. 8. The overall system as the interconnection of two stable subsystems $G_{1}$ and $G_{2}$.

Proof. First we prove properness of $K$. By admissibility we have that $\bar{u}$ is bounded for every $y_{\mathrm{d}} \in H_{2}$. This implies that the input sensitivity matrix $U:=(I-Q+(L+C) P)^{-1}(L+C)$ is a stable transfer matrix. In particular, $U$ is bounded at infinity. Strict properness of $P$ implies that $U(\infty)=K(\infty)$ and hence $K$ is also bounded at infinity (proper). To prove well-posedness, we need to show that $(I+K(\infty) P(\infty))$ is invertible, which is an immediate consequence of the above. To prove internal stability, consider the block diagram depicted in Fig. 7. The dashed box represents the equivalent controller. The shaded box represents the ILC part of the overall system, which we denote as $G_{1}$. The non-ILC part of the system is denoted as $G_{2}$. The respective systems are given as

$G_{1}=\left[\begin{array}{ll}Q & L\end{array}\right]$,

$G_{2}=\left[\begin{array}{c}(I+C P)^{-1} \\ -P(I+C P)^{-1}\end{array}\right]$.

Note that $G_{1}$ and $G_{2}$ are both stable transfer matrices. The overall system can be represented as the feedback interconnection of the subsystems (see Fig. 8). Under these conditions the overall system is internally stable if and only if Zhou et al. (1996, Theorem 5.7)

$\left(I-G_{1} G_{2}\right)^{-1} \in R H_{\infty}$,

where

$\left(I-G_{1} G_{2}\right)^{-1}=\left[I-(Q-L P)(I+C P)^{-1}\right]^{-1}$.

Condition (42) holds by assumption of admissibility (direct implication of Theorem 11). This concludes the proof.

Now we turn to the second question: Is it true that-like in the case of the standard ILC problem-for every stabilizing controller $K \in S$ we can find a corresponding admissible pair
$(Q, L) \in A$ ? The answer is negative. Let $S_{A}$ denote the set of all equivalent controllers. We have the following theorem.

Theorem 13. Given a real-rational plant $P$ and a stabilizing controller $C$. The set $S_{A}$ of all equivalent controllers associated with the family of iterations (5) satisfies the inclusion relation

$S_{A} \subseteq S$

where equality $(=)$ holds if and only if $C$ is a strongly stabilizing controller (that is, if $C$ is both stable and stabilizing).

Proof (Sufficiency). The fact that $S_{A} \subseteq S$ was established in Theorem 12. To prove: $S_{A}=S$ iff $C \in R H_{\infty}$. Suppose $C \in$ $R H_{\infty}$ and let $K$ be any stabilizing controller. Define

$Q:=(K-C)(I+P K)^{-1} P$,

$L:=(K-C)(I+P K)^{-1}$.

Clearly $Q, L \in R H_{\infty}$. Moreover, $(Q-L P)(I+C P)^{-1}=0$, which is sufficient for admissibility (Theorem 11 ). This proves sufficiency.

Proving necessity turns out to be harder. For that we need a few more intermediate results.

Lemma 14. Let $(Q, L)$ be an admissible pair and let $K=(I-$ $Q)^{-1}(L+C)$ denote the corresponding equivalent controller. Then there exists $\left(Q_{0}, L_{0}\right) \in A$ such that $K=\left(I-Q_{0}\right)^{-1}\left(L_{0}+\right.$ C) and $\left(Q_{0}-L_{0} P\right)(I+C P)^{-1}=0$.

Proof. It is clear that there exists $\left(Q_{0}, L_{0}\right) \in A$ such that $\left(Q_{0}, L_{0}\right) \simeq(Q, L)$ if and only if the following set of equations has a solution in $A$ :

$(L+C)=(I-Q) K$,

$(Q-L P)(I+C P)^{-1}=0$.

The unique solution to the above set of equations is given by

$Q_{0}=(K-C) P(I+K P)^{-1}$,

$L_{0}=(K-C)(I+P K)^{-1}$.

From (45) we obtain

$K-C=L+Q K$.

Recall that $Q, L \in R H_{\infty}$ by assumption of admissibility. We substitute (47) into (46) and upon inspection we conclude that $Q_{0}, L_{0} \in R H_{\infty}$. By construction, $\left(Q_{0}, L_{0}\right)$ is admissible. This concludes the proof.

What Lemma 14 says is that again, like in the case of standard ILC, we can restrict attention to a smaller set of representative pairs without giving up generality. We will exploit this fact to characterize the set $S_{A}$. Note that the set $A_{0}$ of all $(Q, L) \in A$ for which $(Q-L P)(I+C P)^{-1}=0$ can be parameterized as follows:

$A_{0}=\left\{(Z N, Z M): Z \in R H_{\infty}\right\}$,

where $P=M^{-1} N$ is a left-coprime factorization over $R H_{\infty}$. This coincides with the parameterization we found for $A_{0}$ in 
the case of standard ILC where we took $M=I$ and $N=P$. Through (48) we arrive at an efficient parameterization of the set $K_{A}$.

Lemma 15. Let $C=V^{-1} U$ and $P=M^{-1} N$ be left-coprime factorizations of the plant and the controller, respectively. Then the set of all equivalent controllers $S_{A}$ is given as

$S_{A}=\left\{(V-V Z M)^{-1}(U+V Z N): Z \in R H_{\infty}\right\}$.

Proof. The equivalent controller is given by

$K=(I-Q)^{-1}(L+C)$.

With $(Q, L) \in A_{0}$ this evaluates to

$$
\begin{aligned}
K & =(I-Z M)^{-1}\left(V^{-1} U+Z N\right) \\
& =(V-V Z M)^{-1}(U+V Z N) .
\end{aligned}
$$

This concludes the proof.

Although the above parameterization seems to depend on specific factorizations, in actual fact the choice of coprime factors is immaterial. This follows from the fact that left-coprime factors are unique up to a left multiplication with a bistable transfer function. The following lemma restates Youla and Kučera's on the parameterization of all stabilizing controllers for a given plant $P$.

Lemma 16 (Youla-Kučera). Given $C=V^{-1} U$ and $P=M^{-1} N$ with $U, V$ and $M, N$ left-coprime. Assume $C$ is stabilizing. Then the set $S$ of all stabilizing controllers is given by Zhou et al. (1996, Theorem 5.7)

$S=\left\{(V-\tilde{Z} M)^{-1}(U+\tilde{Z} N): \tilde{Z} \in R H_{\infty}\right\}$.

Inspection of the respective parameterizations $S_{A}$ (Lemma 15 ) and $S$ (Lemma 16) shows $S_{A}=S$ if and only if for every $\tilde{Z} \in$ $R H_{\infty}$ there exists $Z \in R H_{\infty}$ such that $\tilde{Z}=V Z$. The condition for equality is clearly satisfied in case $C$ is strongly stabilizing $\left(C \in R H_{\infty}\right)$ since then $V$ is bistable and $Z$ can be taken to be $Z=V^{-1} \tilde{Z}$. This agrees with Theorem 13 . We conclude this section with the remaining part of the proof of Theorem 13.

Proof of Theorem 13 (Necessity). We need to show that $S_{A}=S$ only if $C \in R H_{\infty}$. Suppose $C \notin R H_{\infty}$. Take $\tilde{Z}=I$ and let $K$ be the corresponding stabilizing controller (Lemma 16). By uniqueness of the Youla parameter it is clear that the corresponding controller $K$ belongs to $S_{A}$ if and only if there exist $Z \in R H_{\infty}$ such that $V Z=I$. This however implies that $V$ is bistable and $C=V^{-1} U$ stable, which contradicts our starting assumption. This concludes the proof.

Theorem 13 shows that, in general, the set of equivalent controllers constitutes but a subset of all stabilizing controllers. This result suggests that if ILC is designed around a suboptimal feedback controller, it may not be possible to achieve optimal feedback performance in all cases. Our decision to fix the current cycle operator $C$ was motivated by the notion that in practice ILC is more commonly used as an add-on than as a stand-alone control method. If $C$ were a free parameter, it would trivially follow that ILC would do no worse than conventional feedback. Lastly, we remark that there are other CCF configurations of interest. For instance, one could apply standard ILC to the closed-loop system. In that case, the results of Section 3 would apply.

\section{Conclusion}

In this paper we have characterized the set of admissible pairs for a class of linear learning rules. Specifically, for the stable plant case, we have shown that the set of admissible pairs is isomorphic to the set of stabilizing feedback controllers and we have explored the consequences of this result. For the case of an unstable plant under CCF, we showed that the set of admissible pairs is generally smaller than the set of stabilizing controllers. At a more general level, our analysis suggests that, under certain specific conditions detailed in the paper, causal ILC and conventional feedback are truly equivalent methods. If these conditions are not satisfied, equivalence cannot be established. From the results it is clear that causality constrains the application of ILC to a point that one can reasonably question its use. While the strength of ILC is in its potential to exploit information that is inaccessible to conventional controllers, this very potential does not materialize in causal ILC. In view of this we propose that future research should focus on non-causal ILC. The framework developed in this paper can readily be extended to incorporate non-causal operators (Verwoerd, 2005), and it would seem that extensions to nonlinear operators are also possible. Given in particular the body of literature on the Youla parameterization problem for nonlinear systems, it seems reasonable to expect that the correspondence between ILC and conventional feedback control extends beyond the realm of linear systems.

\section{Acknowledgments}

The authors would like to thank the reviewers for their many helpful comments.

\section{References}

Amann, N., Owens, D. H., \& Rogers, E. (1994). New results in iterative learning control. In Proceedings of the IEE Control'94 (pp. 640-645), March.

Amann, N., Owens, D. H., Rogers, E., \& Wahl, A. (1996). An $H_{\infty}$ approach to linear iterative learning control design. International Journal of Adaptive Control and Signal Processing, 10(6), 767-781.

Arimoto, S. (1998). A brief history of iterative learning control. In: Z. Bien, \& J. Xu (Eds.), Iterative learning control: Analysis, design, integration and applications (pp. 3-7). Dordrecht: Kluwer Academic Publishers.

Arimoto, S., Kawamura, S., \& Miyazaki, F. (1984). Bettering operation of robots by learning. Journal of Robotic Systems, 1(2), 123-140.

Chen, Y., \& Wen, C. (1999). Iterative learning control: Convergence, robustness and applications. Lecture notes in control and information sciences. Berlin: Springer.

Goldsmith, P. B. (2001). The fallacy of causal iterative learning control. In Proceedings of the 40th conference on decision and control (pp. 4475-4480), Orlando, FL, USA. 
Goldsmith, P. B. (2002). On the equivalence of causal LTI iterative learning control and feedback control. Automatica, 38(4),

Goldsmith, P. B. (2004). Author's reply to 'comments on 'on the equivalence of causal lit iterative learning control and feedback control". Automatica, 40(5), 899-900.

Kavli, T. (1992). Frequency domain synthesis of trajectory learning controllers for robot manipulators. Journal of Robotic Systems, 9(5), 663-680.

Moore, K. L. (1993). Iterative learning control for deterministic systems. Advances in industrial control. Berlin: Springer.

Moore, K. L. (1999). Iterative learning control—An expository overview. Applied and Computational Controls, Signal Processing, and Circuits, l(1), 151-214.

Moore, K. L., Dahleh, M., \& Bhattacharyya, S. P. (1992). Iterative learning control: A survey and new results. Journal of Robotic Systems, 9(5), 563 -594 .

Owens, D. H., \& Rogers, E. (2004). Comments on 'on the equivalence of causal lti iterative learning control and feedback control'. Automatica, 40(5), 895-898.

Padieu, F., \& Su, R. (1990). An $H_{\infty}$ approach to learning control system. International Journal of Adaptive Control and Signal Processing, 4.

Phan, M. Q., Longman, R. W., \& Moore, K. L. (2000). Unified formulation of linear iterative learning control. Proceedings of AAS/AIAA flight mechanics meeting, clearwater florida.

de Roover, D. (1996). Synthesis of a robust iterative learning controller using an $H_{\infty}$ approach. Proceedings of the 35th conference on decision and control (pp. 3044-3049), December.

Verwoerd, M. H. A. (2005). Iterative learning control: A critical review. Ph.D. thesis, University of Twente, The Netherlands.

Verwoerd, M. H. A., Meinsma, G., \& de Vries, T. J. A. (2002). On the use of noncausal LTI operators in iterative learning control. Proceedings of the 41st conference on decision and control (pp. 3362-3366).

Verwoerd, M. H. A., Meinsma, G., \& de Vries, T. J. A. (2003). On equivalence classes in iterative learning control. Proceedings of the American control conference, Denver, Colorado, USA (pp. 3632-3637).

Xu, J.-X., \& Tan, Y. (2003). Linear and nonlinear iterative learning control. Number 291 in Lecture notes in control and information sciences. Berlin: Springer.

Zames, G. (1981). Feedback and optimal sensitivity: Model reference transformations, multiplicative seminorms and approximate inverses. IEEE Transactions on Automatic Control, 26, 301-320.
Zhou, K., Doyle, J. C., \& Glover, K. (1996). Robust and optimal control. Englewood Cliffs, NJ: Prentice-Hall.

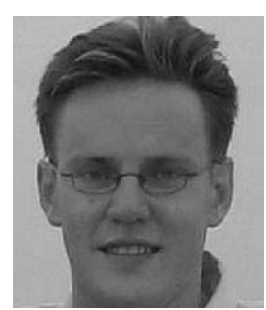

Mark Verwoerd was born in Woerden, The Netherlands, in 1977. He obtained a Ph.D. degree in electrical engineering from the University of Twente, The Netherlands, in 2005. Since March 2005 he is with the Hamilton Institute, at the National University of Ireland. His main interest is in the area of mathematical control theory and its applications in biology.

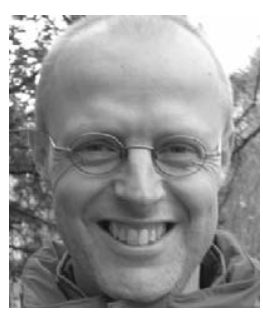

Gjerrit Meinsma was born in Opeinde, The Netherlands, in 1965. He received his Ph.D. degree at the University of Twente in 1993. Over the subsequent three years he held a postdoc position at the University of Newcastle, Australia. Since 1997 he is with the Department of Applied Mathematics at the University of Twente, The Netherlands. His research interests are in mathematical systems and control theory, in particular robust control theory.

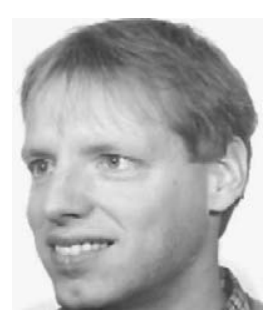

Theo J. A. de Vries was born in Wolvega, The Netherlands, in 1966. He received the M.Sc. and $\mathrm{Ph} . \mathrm{D}$. degrees in electrical engineering from the University of Twente, Enschede, The Netherlands, in 1990 and 1994, respectively, following a special program that combined courses of the Faculties of Electrical Engineering and Mechanical Engineering. From 1994 to 1999, he was an Assistant Professor, and since 1999, he has been an Associate Professor in intelligent control and mechatronics at the Control Laboratory, the University of Twente. In 2001, he cofounded imotec B.V., a mechatronic engineering and development company, and operates as CTO for this company since then. His main research interest is the development of controlled electromechanical systems using learning controllers. 